Musées, Patrimoine et Culture scientifiques et techniques

$111 \mid 2007$

mai - juin 2007

\title{
La gratuité, point aveugle des politiques culturelles
}

\section{Claude Fourteau}

\section{(Q) OpenEdition \\ Journals}

Édition électronique

URL : http://journals.openedition.org/ocim/757

DOI : 10.4000/ocim.757

ISSN : 2108-646X

Éditeur

OCIM

Édition imprimée

Date de publication : 1 mai 2007

Pagination : 15-22

ISSN : 0994-1908

\section{Référence électronique}

Claude Fourteau, "La gratuité, point aveugle des politiques culturelles », La Lettre de l'OCIM [En ligne],

111 | 2007, mis en ligne le 15 mars 2011, consulté le 19 avril 2019. URL : http://

journals.openedition.org/ocim/757 ; DOI : 10.4000/ocim.757 


\section{La gratuité, point aveugle des politiques culturelles}

\section{Claude Fourteau *}

\section{Lơvire,}

\section{Le premier dimanche du mois au musée du Louvre, ce qui n'a pas de prix est gratuit}

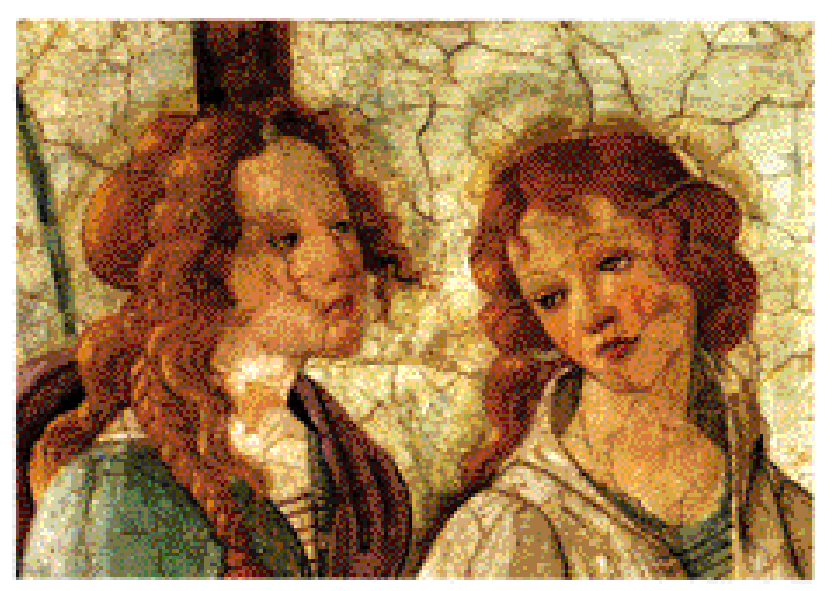

Rapport d'évaluation de la gratuité du dimanche $1996-2000$

* Claude Fourteau a participé à l'ouverture du Centre Pompidou et dirigé le service Liaison/Adhésion de 1981 à 1994. Elle a été responsable de la politique des publics du musée du Louvre et chef adjoint du service culturel de 1994 à 2002, puis chargée de mission auprès de la direction jusqu'en 2004. Elle exerce aujourd'hui une activité d'enseignement et d'expertise sur les questions de publics. fourteau@wanadoo.fr
L'expérience de gratuité le premier dimanche du mois mise en place au musée du Louvre entre 1996 et 2000 a permis de mener pour la première fois une enquête de longue durée sur ce sujet sensible. L'auteur, chargée de cette évaluation, revient ici sur les principaux enseignements de l'expérience et analyse les effets de la gratuité sur le comportement des visiteurs, montrant notamment que pour atteindre l'un de ses buts - l'accessibilité à tous du musée - la gratuité nécessite un accompagnement de médiation.

La passion n'est jamais bien loin dans la controverse qui oppose les tenants de la gratuité à ceux du paiement de l'accès aux biens culturels, et les arguments échangés frappent plus souvent par le poids des convictions que par le recours à la preuve. La faiblesse et la force du sujet résident là, dans l'émotivité qui empreint la problématique du couple gratuité-culture et qui est elle-même à prendre en compte comme un signe de la puissance de son objet et des valeurs qu'il recouvre. Il semble donc utile de préciser la position particulière que l'on assume comme analyste de la gratuité : pour ma part, c'est celle d'un acteur impliqué de l'intérieur dans la politique des publics de musées, ayant abordé depuis longtemps la question des tarifs par le biais d'une grande diversité d'expériences de terrain et de travaux, mais toujours à partir du public et de la problématique de l'accès et de l'éducation. La question tarifaire dans les musées et monuments était jusqu'à récemment laissée au soin des gestionnaires. Aussi est-ce une grande avancée 
qu'à la suite d'un retour de la gratuité du dimanche au Louvre en 1996, ce débat ait repris place dans l'espace public, où il y a seulement dix ans régnait une sorte d'omerta.

Depuis le tournant des années 2000, la gratuité n'a cessé de se propager ; les pratiques culturelles sont devenues un enjeu d'offres gratuites disparates au point d'appeler la question : parle-t-on d'une même notion ? Il nous a paru important de réfléchir à ce que la gratuité a de spécifique lorsqu'elle s'exerce dans le domaine du patrimoine et, dans le prolongement des études que nous avons précédemment menées sur la fonction attribuée historiquement à la gratuité, sur ses évolutions et sur son évaluation au Louvre et dans d'autres institutions culturelles (1), de proposer un modèle théorique portant sur la façon dont elle est vécue aujourd'hui par les visiteurs de musées. Bien que le Louvre ait un statut d'exception de par sa taille, sa notoriété, son épaisseur historique, il apparaît cependant que cet excès d'échelle et de sens favorise l'expression des réactions du public et fait de ce musée un paradigme des lieux de patrimoine en France. Limpact de la réintroduction de la gratuité a d'ailleurs été sensiblement parallèle au Louvre et dans les autres musées et monuments.

\section{L'évaluation de la gratuité d'un dimanche par mois au Louvre}

Pour commencer cette analyse, il faut donc rappeler brièvement le contexte du réveil de la gratuité, après une période d'abolition dans les musées nationaux. La responsabilité a été confiée au Louvre de réinstruire le dossier de la gratuité en 1995, à la demande du ministre de la Culture désireux d'expérimenter pendant deux ans et d'évaluer l'impact de la réintroduction de la gratuité le premier dimanche de chaque mois. La détermination de la direction du Louvre à favoriser les conditions d'une étude à très grande échelle, durable, entourée de toutes les garanties scientifiques nécessaires, a permis au

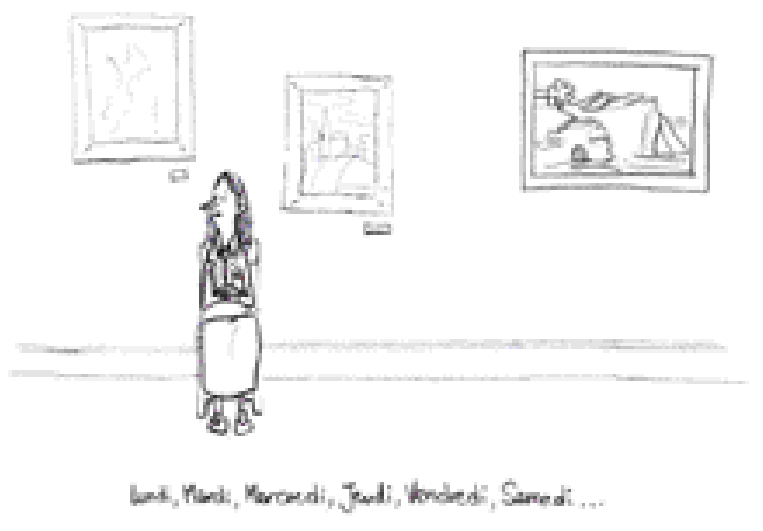

ministère, au vu de l'évaluation, de transformer l'expérimentation en régime définitif, en étendant en 2000 la gratuité d'un dimanche par mois à tous les musées nationaux et aux monuments historiques. Pour nous, en charge du pilotage de l'évaluation du Louvre ${ }^{(2)}$, la gratuité fut un combat pour comprendre, sur un terrain quasiment vierge d'études mais fortement investi d'idéologie. Quel est l'effet produit sur les visiteurs et sur la visite lorsqu'est réintroduite la gratuité ? Cette question simple nous a amenés à construire une méthodologie d'enquête largement inusitée, que nous précisons ici pour que le lecteur se repère dans la diversité des travaux qui se sont ensuite multipliés sur la question.

Nous souhaitions d'une part apporter pour la première fois une information incontestable sur la stricte mesure quantifiée de l'impact du retour de la gratuité. La chance nous était donnée de travailler non sur des projections prédictives, mais sur une situation réelle, en étudiant ceux-là mêmes sur qui la gratuité est expérimentée, c'est-à-dire les visiteurs, plutôt que d'effectuer des sondages sur échantillons représentatifs de la population dans son ensemble, ce qui a pour résultat de noyer les effets, et généralement de les invalider ; la chance aussi de profiter de l'immense audience diversifiée de l'institution la plus fréquentée au monde pour donner une dimension massive à l'enquête (23 600 questionnaires traités, en 9 langues, recueillis par moitié les dimanches payants, par moitié les dimanches gratuits), ce qui nous a permis d'affiner la connaissance jusqu'à un niveau de variables très détaillé - croisements qui seraient impossibles sur les petits échantillons - ; d'intégrer la population étrangère, largement majoritaire dans les plus grands musées et généralement absente des enquêtes, alors que ses réactions à la gratuité constituent un élément de poids essentiel au débat ; de travailler sur des nombres réels, les visiteurs étant systématiquement comptés à l'entrée, au lieu de s'en tenir aux seuls pourcentages, qui ignorent et écrasent les quantités ;

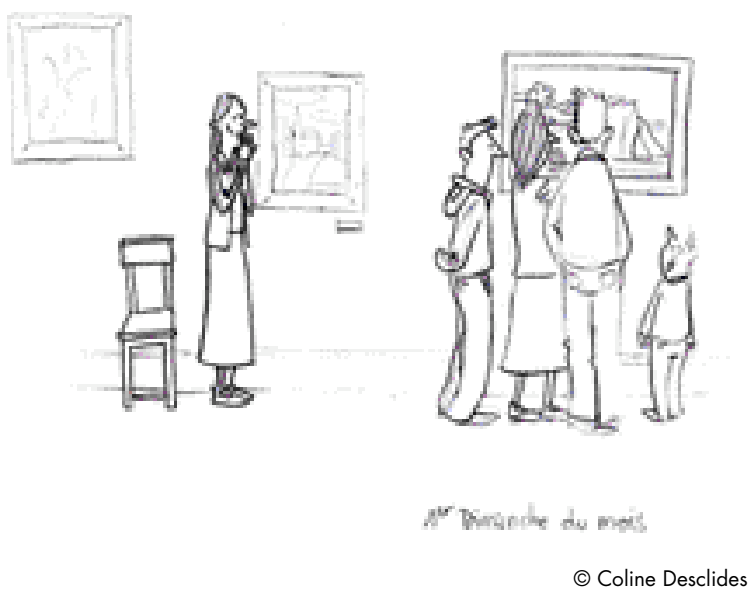


enfin, de prendre le temps de poursuivre l'enquête, en allongeant la durée de l'évaluation de deux à cinq ans pour permettre une analyse de l'évolution des effets dans le temps.

L'écart des résultats entre dimanches payants et gratuits s'est révélé considérable, au point de démontrer que la gratuité est sans conteste l'instrument le plus puissant qui soit à la disposition des politiques culturelles. Les principaux résultats sont les suivants : la gratuité entrâne un accroissement de fréquentation massif et durable (le nombre total de visiteurs croît de $60 \%$ le dimanche gratuit) ; l'ancrage national du musée s'en trouve considérablement amplifié (les Français ou résidents en France sont 2,3 fois plus nombreux) ; l'incitation à la visite, en particulier à la première visite, est l'effet majeur de la gratuité (45\% des visiteurs français informés de la gratuité déclarent qu'ils ne seraient pas venus sans cette mesure) ; toutes les catégories socioprofessionnelles et tous les âges sont concernés, et à l'intérieur de cet effet général, tous les indicateurs de démocratisation sont positifs (le nombre des visiteurs franciliens issus des catégories supérieures est multiplié par 2,6; celui des professions intermédiaires par 2,8; celui des employés/ouvriers par 3,1 ; les étudiants par 4,5; les familles par 3,4). Enfin, la gratuité fidélise : 1/3 des Franciliens interrogés un dimanche gratuit sont déjà venus un dimanche gratuit précédent.

\section{Le mode opératoire de la gratuité}

Au-delà des chiffres, nous souhaitions entrer dans une démarche de compréhension de la perception de la gratuité, des motivations des visiteurs et de la conduite de leur visite. Ce deuxième volet, confié à Hana Gottesdiener (3), a ouvert le champ d'une analyse qualitative par entretiens approfondis, individuels ou en groupes, extrêmement éclairante sur le mystère des causes de l'ampleur des résultats livrés par l'étude quantitative. Afin de revenir sur les processus qui rendent la gratuité opérante, nous nous fonderons sur la réflexion que les acteurs eux-mêmes portent sur leur expérience et sur l'interprétation déjà réalisée dans l'étude précitée. Tous les verbatim cités dans ce texte en proviennent et sont mentionnés en italiques.

Ces processus, au cours desquels se succèdent des étapes de "formation d'un visiteur en devenir " (Gottesdiener), reposent sur une suite d'articulations dialectiques qui fonctionnent autour de la gratuité par couples de concepts : l'exemption du prix et la liberté, le don et la dette, le droit à la culture et le dû.
Pour fonder ce schéma en théorie, c'est vers la fameuse " énigme du don », qui a tant occupé les anthropologues, qu'il nous a semblé naturel de nous tourner pour y confronter l'énigme de la gratuité, et nous avons interrogé tout particulièrement l'analyse des trois modes de " circulation des objets » qui existent dans toutes les sociétés : ceux qu'on vend, ceux qu'on donne, ceux qu'on transmet (4) et (5). En développant les voies ainsi ouvertes, nous allons tenter de suivre les interférences et les réactions que suscite la gratuité dans chacun de ces systèmes.

\section{L'exception / I'occasion : \\ la gratuité dans la sphère du marché}

Un premier mode d'échange est celui des objets ou services que l'on vend et que l'on achète sur les marchés. La visite payante est devenue la norme dans les musées et monuments - tandis que la gratuité demeurait la norme dans les bibliothèques. Peutêtre n'a-t-on pas mesuré à quel point l'introduction de la tarification a bouleversé les logiques
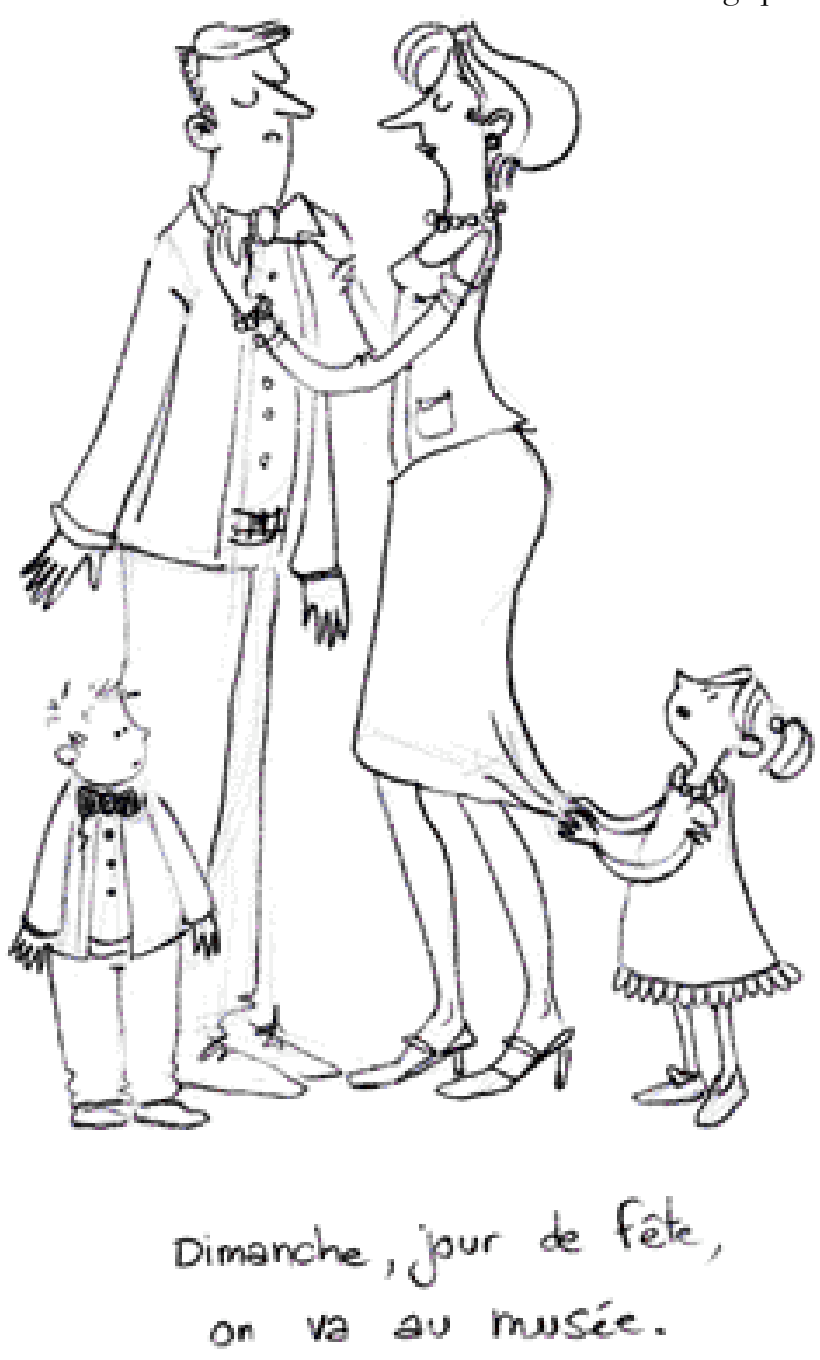

(C) Coline Desclides 
des institutions patrimoniales et introduit d'ambiguïté dans le registre des valeurs qui sous-tendent leur existence, au fur et à mesure que les plages de gratuité pour tous se réduisaient et finalement disparaissaient. L'offre publique, telle qu'elle-même se communique aujourd'hui, ne se distingue plus guère de l'offre marchande, ni la pratique culturelle de la consommation. L'échange tarifé a permis d'engager une dynamique de développement riche d'efficacité et pauvre de contenu relationnel, car il produit une relation fermée, qui se résout dans l'instant du donnant-payant, au cours de laquelle le bien est aliéné par l'acheteur : " quand j'ai payé, je veux un service et puis voilà ».

Comment la gratuité peut-elle trouver place dans le système marchand ? On l'y inscrit tout simplement à l'intérieur de la politique tarifaire, en tant que catégorie d'exception. C'est le tarif à prix zéro. Elle se manifeste de deux manières, soit comme exonération catégorielle et elle vise des populations ciblées, soit comme exemption générale liée à des événements, gratuité d'un jour ou d'une nuit blanche, qui produit un effet d'annonce en faveur du musée et constitue un produit d'appel pour la visite. Dans les deux cas, le terme de gratuité est ambigu, puisqu'il ne s'inscrit que comme exception à un tout payant. On ne saurait mieux l'illustrer que dans l'exemple de ce projet d'affiche proposé par un publicitaire pour annoncer la gratuité d'un dimanche par mois : « Le Louvre à zéro franc ! ».

Le triptyque des arguments en faveur du paiement n'a guère changé depuis plus d'un siècle : le paiement à l'entrée du musée sélectionne le public, protège les œuvres, enrichit l'institution (6) (7) et (8). Le paiement sert en effet à se prémunir des importuns qui n'ont pas leur place dans les musées : «n'importe qui rentre dans le musée ». Dans un monde où le prix est l'étalon de la valeur, le paiement imposerait le respect pour les œuvres alors que la gratuité les dévaloriserait : "on n'a plus la notion de ce qu'on va voir "; "les gens viennent au musée comme s'ils faisaient une balade dans une grande surface ». En réduisant le nombre des visiteurs, l'entrée payante permet de limiter la foule, le bruit, l'attente. Enfin, elle donne au musée des ressources précieuses et au public payant le sentiment de participer au financement du musée. La gratuité en revanche, contestée dans son efficacité sociale et toujours vue en creux, produirait surtout du manque à gagner dû aux «effets d'aubaine ", qui attireraient au musée majoritairement ceux qui auraient les moyens de payer et qui seraient ainsi des « profiteurs » qui «abusent». Quant aux visiteurs impécunieux - les seuls que viserait la gratuité - soit ils ne viendraient pas, soit ils seraient pour l'essentiel dépourvus du niveau de culture nécessaire pour tirer profit de la visite. On observe que lorsque ces arguments, fréquemment utilisés par des responsables culturels ou des sociologues, se trouvent partagés par des visiteurs, il s'agit dans la plupart des cas de visiteurs payants.

Les visiteurs du dimanche gratuit en revanche, vivent les choses bien différemment. D’une part ils sont bien là, en grand nombre, beaucoup venant pour la première fois, et l'on ne peut que s'étonner de constater que les preuves pourtant irréfutables des effets de renouvellement et de démocratisation de cette journée restent encore si souvent méconnues ou déniées. Ces nouveaux visiteurs n'hésitent pas à reconnaître que la journée de gratuité a constitué pour eux "l'occasion", "le plus qui donne envie d'y aller ", " le pied à l'étrier ». Il est très intéressant de s'arrêter au récit de leur expérience, qui jette un jour nouveau sur le rapport de la population à la culture et à l'argent.

La première vague de témoignages rend compte de la réalité de la « barrière » du paiement et exprime le sentiment de pénurie financière : "c'est très cher d'aller au musée, c'est très cher de se cultiver " (une vendeuse); "une famille qui arrive à quatre personnes, plus le déplacement, ça devient très cher » (un électricien) ; "on se dit ça ferait plaisir d'aller pour une heure, mais une heure à trois, ça fait très cher » (une femme avec enfants) ; "l'espace culture dans une famille avec trois enfants est très restreint » (femme de commerçant). Notons que, s'agissant de personnes qui déclarent être venues au musée ce jour-là parce qu'il était gratuit, le recours à l'analyse classique selon laquelle l'argument financier serait un alibi pour couvrir le manque d'intérêt culturel est caduc. Une visiteuse, intendante de profession, se charge de faire l'analyse économique : "le prix des musées et des expos est devenu trop cô̂teux. Il s'est croisé une augmentation des prix avec une politique commerciale, ça fait que les prix sont importants au moment même où le pouvoir d'achat des gens s'est mis à baisser ».

Si la gratuité d'un jour paraît dans ce contexte économique difficile comme "une chance », la capacité d'entrer sans payer ne suffit pas pour rendre compte de la décision de visite. Le plaisir palpable qui rend la visite attrayante et festive est celui d'être libéré du poids de l'argent : "le fait que ce soit gratuit ça aide, parce qu'on n'a pas à se soucier» (un étudiant). C'est 


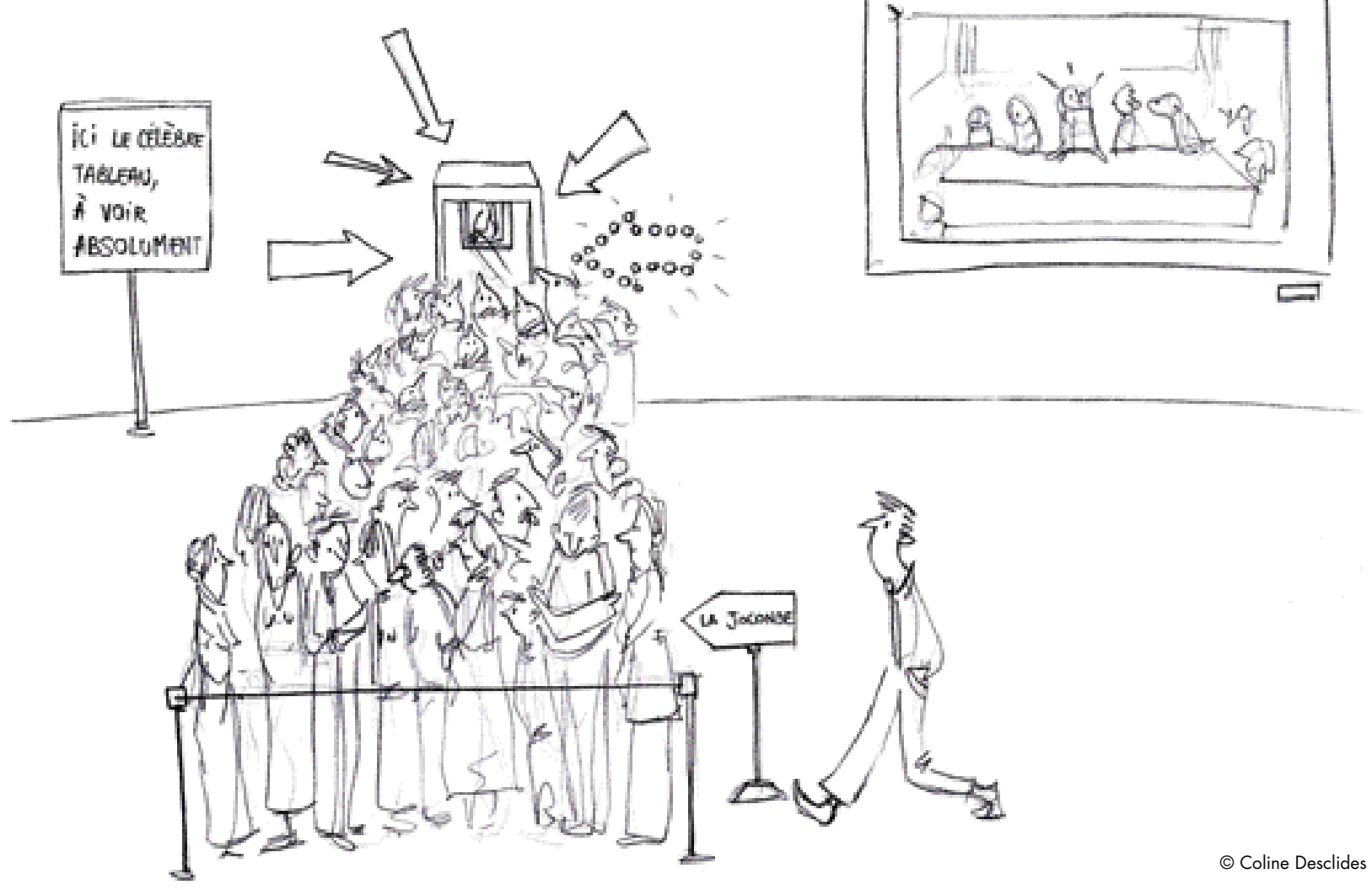

qu'une forte contrainte morale est attachée à l'argent : celle de justifier sa dépense. La description que font les visiteurs de la visite payante est de ce point de vue saisissante : « ...payer, et en retour il faut voir, voir, voir...» (conducteur de travaux) ; «je sais que c'est de l'argent que j'investis. Je ne pense même pas que j'aurai le temps de m'asseoir, je voudrais tout voir » (une étudiante).

L'on voit bien ici que " l'effet d'aubaine » si souvent évoqué n'a de pertinence que lorsqu'il est appliqué à ceux qui font commerce du droit d'entrée, c'est-àdire les opérateurs du tourisme - et il suffit d'interdire la visite en groupe les jours de gratuité pour limiter ce problème. En revanche, le visiteur ordinaire exprime une forte sensibilité au prix (en particulier les catégories populaires, les familles et les étudiants) et plus encore une sensibilité à la valeur de l'argent (très largement partagée dans toutes les catégories sociales). Fortement intériorisée, une morale de la dépense entre en balance avec l'injonction à s'instruire : quand elle n'est pas un frein à la visite, elle fait un devoir au visiteur nouveau ou occasionnel de s'imposer un parcours du musée accumulant le nombre d'œuvres, la distance parcourue, le temps passé, la fatigue. On en conclura que pour les personnes à faibles revenus ou faible pratique du musée le paiement est un obstacle sérieux à la visite, et que pour la majorité de la population le paiement est un obstacle majeur à la qualité et au plaisir de la visite, et en particulier des premières visites, qui conditionnent l'envie de retour.

Délivrée de «l'astreinte » du paiement, la visite gratuite en revanche ouvre à des dispositions qui autorisent le plaisir, le choix personnel, l'expression de préférences. C'est alors que l'on peut «être plus détendu » (médecin), "regarder ce qu'on a envie de voir » (cadre commercial), " perdre son temps ", " se faire plaisir » (étudiant), «être plus libre » (gérante d'immeuble). Derrière la gratuité l'on trouve la notion de liberté, le langage confondant d'ailleurs l'expression de l'une et l'autre dans un même adjectif : accès libre, free access, le "Louvre libre », comme titrait Clémenceau dans son plaidoyer contre le paiement ${ }^{(9)}$. La gratuité initie à une grande leçon que l'école et les musées ne pensent pas à transmettre ou souvent même contredisent : la valeur de la pratique culturelle ne se mesure pas en quantité ou en épuisante consommation du voir ; la visite au musée, lorsqu'elle est libre, est un exercice de découverte, de plaisir, parfois de révélation, non pas seulement de la vision d'une œuvre, mais de l'expérience de regarder. Dans la bouche des visiteurs du dimanche, les formules abondent pour rendre compte de cette perception nouvelle du regard : "voir sans voir... on a envie de revenir pour voir ce qu'on a vu... voir de l'intérieur » ; «moi, en 
sortant du Louvre, j'ai téléphoné à mes parents, j'avais l'impression d'avoir quatre ans "; "se laisser aller à regarder»; " et on voit tout le bonheur de regarder». Dans un univers marchand, la gratuité est vécue avant tout comme «libératoire »(Gottesdiener).

\section{La reconnaissance et la dette :}

la gratuité dans la sphère du don

"La question du don, c'est la question des modes de circulation d'objets ou de services qui ne passent pas par le marché " (10).

Lorsque l'État décide de rendre à la gratuité les musées et monuments nationaux un dimanche par mois, il entend adresser « un signe fort pour faire comprendre que la culture s'adresse à tous »(P. Douste Blazy, 1995). Quand ce signe a pour destinataire la collectivité nationale, pour objet la culture et pour objectif l'égalité, il entre dans le cadre distinctif d'une politique publique, qui agit par le moyen de ses propres codes de référence. La gratuité consacre la volonté publique comme un don. Le don est un puissant agent de mise en œuvre d'une relation car il est un appel fait à la population tout entière à se sentir désignée comme donataire. Les anthropologues qui ont étudié la circulation du don dans les sociétés primitives l'analysent comme la reproduction d'une relation de reconnaissance, de subordination et de dette, une relation qui ne cesse jamais de lier le donataire au donateur et à conférer à ce dernier du pouvoir. Contrairement à l'objet acheté, qui est détaché de son propriétaire, l'objet donné continue à lui être attaché par le sentiment de dette de celui qui l'a reçu et qui ne peut espérer équilibrer l'échange que par un contre-don de même valeur ${ }^{(4)}$.

Ce schéma a-t-il à voir avec les réactions des visiteurs que nous avons étudiés, qui ont, rappelons-le, répondu à la gratuité par leur présence au musée, c'est-à-dire qui s'en reconnaissent comme destinataires ? Leur réaction initiale est le plaisir à recevoir une invitation accueillante, qui appelle en retour le geste de la venue. L'acceptation est facilitée du fait que l'invitation s'adresse à tous et quil y aura foule : "ce qui m'a touchée, c'est que j'avais l'impression qu'on me faisait un cadeau, à moi et à des milliers de gens ». Les visiteurs ne sont plus en face d'une occasion à saisir individuellement, ils sont d'emblée projetés dans un espace collectif d'échange non marchand. L'affiche choisie pour l'illustrer est déployée chaque mois dans le métro et appelle le passant à s'attarder sur cette formule énigmatique : "Le premier dimanche du mois, au musée du Louvre, ce qui n'a pas de prix est gratuit».

Le don appelle de fait une double forme de reconnaissance : la gratitude envers le donateur conjuguée

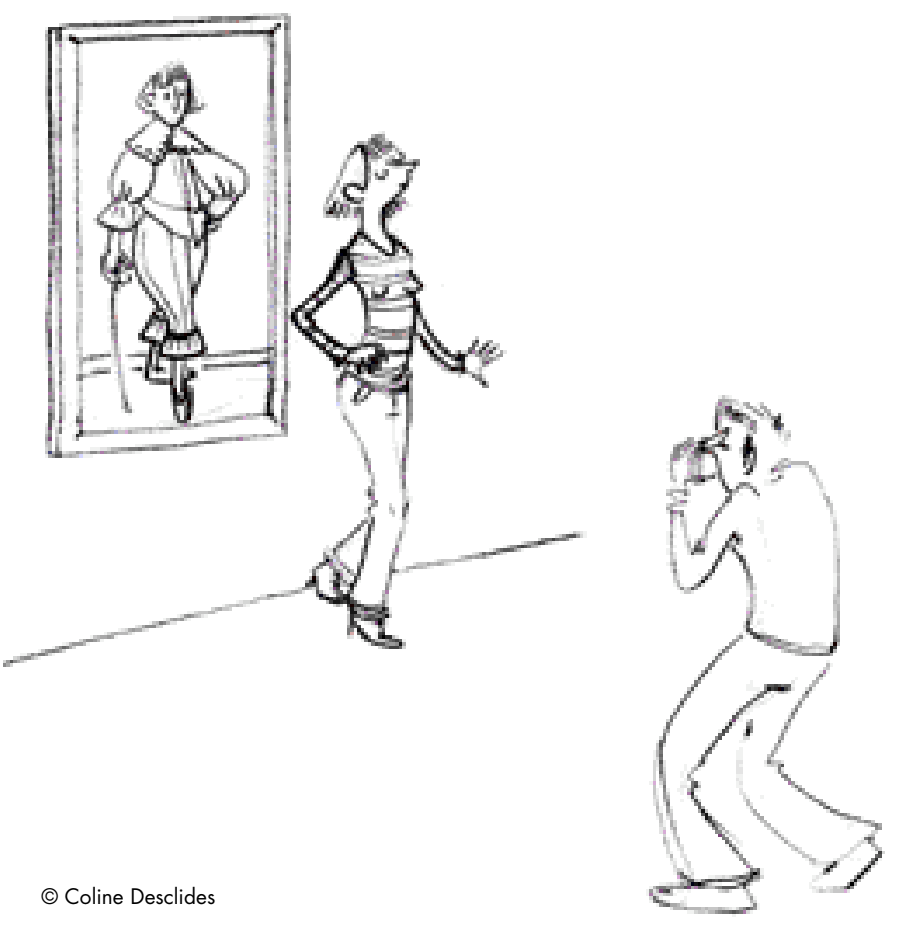

avec la reconnaissance de la valeur de l'objet offert. Il tire sa force de l'assentiment du donataire à le reconnaître pour un bienfait, qui l'oblige : "vous venez nous offrir la possibilité de nous cultiver gratuitement... on est obligés d'y aller ». "Y aller », c'est accepter l'effort de se dégager des facilités et des soucis de la vie courante, des «horreurs » que l'on regarde à la télévision, de "la société où l'on montre que de la violence ", des films de guerre au cinéma, et «si on veut les voir, c'est notre problème». Face à cela - que l'on admet être le laisser-aller ordinaire l'on accepte le pouvoir de la puissance publique et de l'institution culturelle à exercer la fonction de nous rappeler au vrai sens des valeurs. " on se dit, oui, il y a autre chose dans la vie "; "la culture, c'est important parce que... on a tendance à retourner facilement à la barbarie »; "ça humanise, le musée » ; " la culture, c'est le moyen d'expression de l'homme ».

La mesure de gratuité que le politique a voulu expérimenter au musée un dimanche par mois a la particularité de s'inscrire dans la durée, mais non dans la permanence, équilibre que les visiteurs reconnaissent comme particulièrement efficace. À la fois exceptionnelle et régulière, chaque mois la gratuité sonne comme un appel : elle stimule la visite et fidélise le visiteur. Le projet de retour lui ouvre l'horizon d'un projet à construire. Il sait bien que «se cultiver » est un processus qui demande du temps, et ce temps lui est justement offert sous forme de rendez-vous mensuel : "si le système reste gratuit, il est évident qu'on va revenir... et là on va apprendre » 
" un dimanche par mois, on y pense ", " et puis on se prépare ", " ça donne un point de référence ", "ça demande une démarche ", "c'est vrai que c'est un appel ». Ainsi, on retrouve là l'enchaînement des obligations du don, de l'acceptation et de la dette qui va pousser à répondre par un contre-don de même valeur, sous la forme de la décision de s'instruire : "ça me donne du travail pour au moins huit visites ", "ça m'emmène jusqu'au mois d'août ». Un enchaînement vertueux, un contrat personnel organisent le désir du visiteur de s’insérer, par l'effort et le mérite, dans la hiérarchie des valeurs que le musée recèle, fondées sur le savoir. On rencontre là une configuration remarquable des effets de la gratuité comme don, qui provoque la mise en route du désir d'accession à la culture, décision consentie, intériorisée, ouvrant un horizon de progrès personnel et s'appuyant sur une méthode d'approche : la venue régulière et studieuse au musée. Reste, guide à la main et bonne volonté comme bagage, à aborder l'immense labyrinthe des collections, de l'histoire de l'art, du temps des civilisations mortes. Et là, confrontés à la difficulté de la visite et à un apprentissage solitaire, les visiteurs ne manquent pas de s'étonner : le musée souhaite-t-il vraiment favoriser leur progression de « visiteurs en devenir » ? Il ne suffit pas de franchir l'entrée pour accéder aux œuvres. «On est un peu désorientés » ; «ça doit être possible de faire ça, oui expliquer un petit peu la signification de chaque cuvre d'art? " "quand je vais dans un musée, j'aime bien me dire, jy vais, je vais apprendre... mais le vrai sens de l'objet en lui-même, c'est vrai on peut pas le deviner, on perd beaucoup "; "c'est quand même un grand musée, je m'attendais à ce qu'il y ait, quand même ... on a vu beaucoup de gens perdus » ; "il $y$ a rien $d u$ tout, il y a un auteur et un titre... ».

En conclusion de cette étape, deux réflexions se répondent en écho, dans un jeu de vocabulaire qui illustre bien la subtile instabilité de l'échange : «le don n'est jamais gratuit », affirment les anthropologues. Tandis que les visiteurs concluent pensivement : "c'est gratuit, c'est pas donné ». Le don ne serait-il que le moyen, de la part du donateur, de «se positionner dans la célébration de soi »? (10).

\section{L'égalité de droit et le dû :}

la gratuité dans la sphère du symbolique

Il existe une troisième forme d'échange, analysée par Maurice Godelier, auteur de L'Énigme du don (5), comme celle «des objets qui ne peuvent être ni vendus ni donnés, mais qui sont gardés pour être transmis de génération en génération. Ce sont les objets sacrés ». Ces objets énigmatiques, dont on ne transmet jamais la propriété car ils sont collectifs et inaliénables, ne

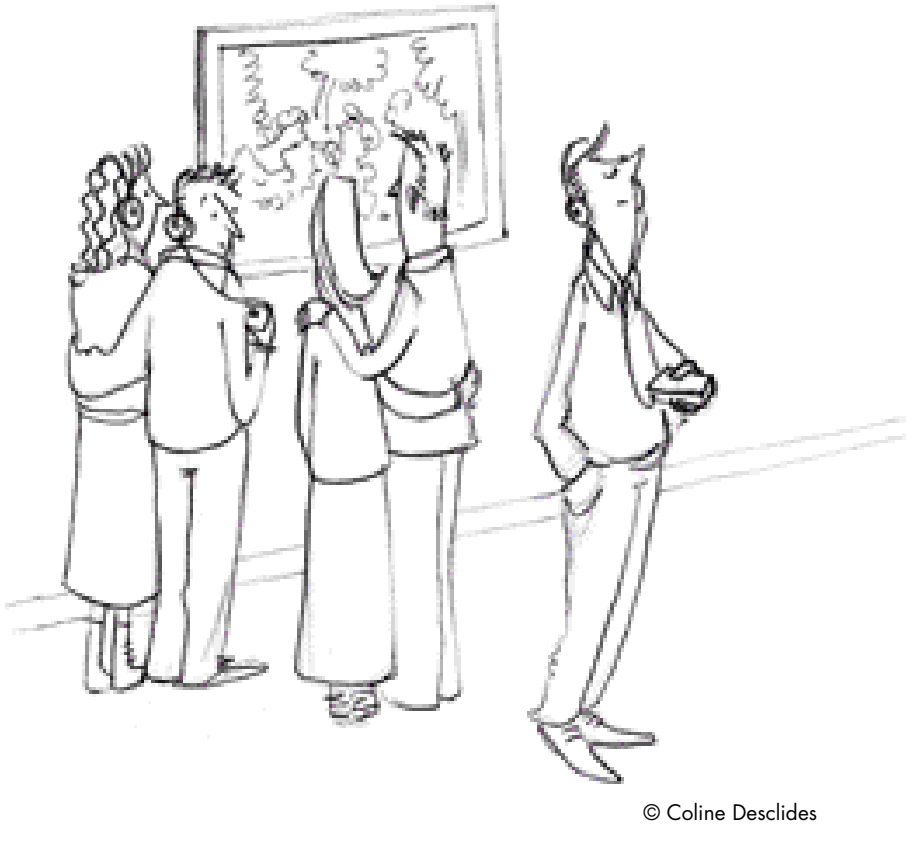

peuvent être offerts qu' "en connaissance et en jouissance », cette forme particulière du don par transmission étant la seule qui soit véritablement gratuite. N'est-ce pas là la caractéristique des objets de musées ? L'anthropologue a lui-même accepté, au cours d'une intervention très éclairante, de valider ce « rapprochement audacieux » entre les rituels qui entourent les objets sacrés dans les sociétés archaïques et l'imaginaire des sociétés modernes à l'égard des objets patrimoniaux ${ }^{(10)}$.

La gratuité, du fait qu'elle soit en débat et qu'elle agisse sur les comportements, incite les visiteurs à faire sur eux-mêmes un travail d'interprétation sur le sens qu'ils lui donnent, et à se prêter en particulier à une élucidation collective au cours des entretiens de groupe où ils s'interrogent : qu'avons-nous en commun dans notre rapport à la gratuité ? La foule qui se presse au Louvre le dimanche gratuit donne à cette question une réalité palpable : nul ne peut s’y méprendre, ce public est essentiellement distinct de celui de tous les autres jours, d'abord du fait du nombre, plus que de moitié supérieur aux autres dimanches, mais aussi de la composition des visiteurs : le dimanche gratuit est le seul jour du mois où le public national est majoritaire au Louvre. Les visiteurs s'étonnent euxmêmes d'y voir " des gens de toutes catégories sociales ", "des jeunes de banlieue, même des provinciaux, des gens avec des petits bébés, des jeunes »... Dans cette foule, le visiteur se voit représenté, lui et tous les autres, et il se réjouit de s'y fondre. Au cours de cette journée particulière, du seul effet de la gratuité, une célébration muette s'opère : la foule est peuple, dans un sentiment de communion semblable à celui 
du « public rassemblé » du théâtre, unie par la mise en suspens des inégalités. Ces jours-là, les notions de droit et d'égalité d'accès à la culture prennent corps. Au cours de la discussion, ces notions font leur chemin dans les esprits, passionnent le débat, la revendication de ce qui est dû au citoyen s'y fait jour : " c'est normal que tout le monde puisse y accéder »; "ça met tout le monde au même niveau, les hauts dirigeants et puis... et nous "; "ça devrait être gratuit, comme à la Révolution ", " il y a ceux qui auront la connaissance et ceux qui n'y auront jamais accès, et qui se sentiront... laissés pour compte "; il faut " donner à tout le monde la possibilité d'avoir accès à l'art et de powvoir évoluer dans l'apprentissage de la culture ». Si les sentiments et les principes sont aussi fortement mobilisés, c'est que le Louvre n'est pas neutre. Ancien Régime et Révolution, palais et musée s'y succèdent, l'histoire de France et les trésors de l'art s'y côtoient. Pour l'ensemble des visiteurs, le Louvre est le symbole de la France et de la culture, le lieu de transmission par excellence où se cristallise lidentité nationale, mais aussi le lieu de l'abolition des privilèges. La soif d'égalité recouvre la revendication d'un patrimoine, d'une histoire, d'un héritage qui appartiennent à tous les Français (3) et qui fonde sa légitimité sur la Révolution et les conquêtes de "nos aïeux ». L'émotivité liée à ces évocations est extrême : " avoir le sentiment... qu'on profite un petit peu de ce qui nous appartient »; « allez-y, vous les verrez vos racines, vous verrez que vous en avez et que la France ne s'est pas construite du jour au lendemain ! "; " on peut très bien reconnaître la valeur d'une ceuvre parce que de toute façon on se l'est déjà appropriée historiquement "; "ça permet aux gens un petit peu de sentir quils appartiennent à une nation ».

Ce qui se joue là est un phénomène qui engage l'imaginaire collectif, à partir d'un grand récit des origines, des conquêtes politiques qui ont créé les fondements de notre contrat social, des aïeux qui nous les ont transmis, et la gratuité est le symbole qui réactualise et revigore les principes de la société liée dont nous sommes. Ce sont eux les objets sacrés, " porteurs de valeurs et d'identités dont on veut assurer la continuité et le partage par des générations successives »(10).

Au terme de cet essai pour comprendre, nous devons admettre que la question posée des effets de la gratuité ne peut être simplement abordée comme l'analyse de l'influence des dispositifs institutionnels sur le comportement et les représentations du public. La gratuité dépasse largement les problèmes de stratégie institutionnelle, au point de renverser l'ordre des facteurs : c'est la gratuité souveraine, point aveugle des politiques culturelles, qui impose au musée de jouer " le rôle d'unification sociale et politique qui lui incombe historiquement " (11). Il en résulte pour les responsables, un devoir de cohérence générale obéissant à des valeurs, qui est une éthique de gouvernance. "Dans une société démocratique, c'est l'équivalent d'une réalité sacrée partagée dont il nous faut assurer l'exercice " (10). Ce serait manquer gravement aux attentes sociales que d'utiliser ces références comme simple rhétorique.

Dans sa mise en pratique, la gratuité requiert-elle l'apanage de l'exclusivité ? L'expérience et le volontarisme invitent à accomplir les principes et à éviter le dogmatisme. Les visiteurs du dimanche pour leur part estiment que la gratuité a plus d'impact sur eux du fait d'être discontinue, pourvu qu'elle soit régulière. Ils admettent aussi que le musée a besoin de financement et sont prêts à y participer pour autant qu'ils le puissent. En revanche, ils demandent instamment du respect, qui se marquerait par un accompagnement de médiation, largement à inventer, qui leur rende le musée accessible. Car la gratuité n'est pas une molle facilité qui exonèrerait le musée de toute autre action culturelle ; elle est exigence accrue d'un engagement actif aux côtés des visiteurs en devenir.

Le dossier d'évaluation de la gratuité du dimanche au Louvre 1996-2000 est disponible sur demande : fourteau@wanadoo.fr

\section{Notes}

(1) Fourteau, C. (dir.) Signe et symbole, l'exemple de la gratuité, textes de Anne Gombault, Sylvia Lahav, Florence Lévy et Katia Papaspiliopoulos, Claude Fourteau, Hana Gottesdiener, Maurice Godelier, pp. 63-143, in Les Institutions culturelles au plus près $d u$ public. Paris : La Documentation française/musée du Louvre, 2002, 280 p.

(2) Fourteau, C. Rapport d'évaluation de la gratuité du dimanche au Louvre, 1996-2000. Paris : musée du Louvre, 2002, 110 p.

(3) Gottesdiener, H. et Godrèche, N. Perceptions et comportements de visite lors des dimanches gratuits au musée du Louvre, in supra, pp. 39-109. (4) Mauss, M. Essai sur le don. Forme et raison de l'échange dans les sociétés archaïques, in Sociologie et Anthropologie. Paris : PUF, 1950.

(5) Godelier, M. L'Énigme du don. Paris : Fayard, 1996, 297 p.

(6) Samsoën, D. Petite histoire de la gratuité dans les musées, in Rouet, F. (dir.) Les tarifs de la culture. Paris : La Documentation française, 2002.

(7) Genermont, D. Historique de la tarification, in Fourteau, C. (dir.) Politiques tarifaires musées et monuments. Paris : Association InterMusées, 1997, 174 p.

(8) Galard, J. Visiteurs du Louvre, un florilège, pp.31-52. Paris : RMN, 1992, 202 p.

(9) Clémenceau, G. Le Louvre libre. 1896.

(10) Godelier, M. L'énigme du don et la présence des objets dans les musées, in Fourteau, C. Les Institutions culturelles au plus près du public. Paris : La Documentation française/musée du Louvre, 2002, pp. 135-143. (11) Poulot, D. Patrimoine et musées, l'institution de la culture. Paris : Hachette, 2001. 